Tropfen Salzsäure bis zum Sieden erhitzt, um das überschüssige unterschwefligsaure Natron zu zerstören, filtrirt, und aus dem Filtrat die Schwefelsäure als schwefelsaurer Baryt bestimmt.

1 Aeq. Schwefelsäure entspricht 2 Aeq. Chlor. Gefunden wurden 0,074 Grm. Schwefelsäure. Diese entsprechen 0,1325 Chlor. Die Unze Chlorwasser enthielt demnach 2,12 Gran Chlor.

Drei andere Bestimmungen gaben dieselben Zahlen.

\title{
Ueber einige Benzoylverbindungen;
}

$$
\text { von Dr. Carl Voit. }
$$

Die Chemiker, welche sich bisher mit der Untersuchung der Benzaminsäure beschäftigten, liefsen ihre Verbindungen mit Basen fast unberücksichtigt und nur das Silbersalz ist von Chancel analysirt worden. Einige Verbindungen der Benzaminsäure mit Säuren, in welchen sie die Rolle einer Basis spielt, studirte Gerland. - Es war ferner das Verhalten des Schwefelwasserstoffs gegen Binitrobenzoësäure noch unbekannt und es liels sich nicht im Voraus bestimmen, ob beide oder nur ein Aequivalent Untersalpetersäure vom Amid substituirt würden. - Auf Veranlassung des Herrn Professors Limpricht versuchte ich, diese Lücken auszufüllen.

Ehe ich zur Beschreibung meiner Versuche übergehe, will ich hier auf den unpassenden Namen „Benzaminsäure (und Anisamiusäure)“ aufmerksam machen. Bekanntlich können nur die Radicale zweibasischer Säuren Aminsäuren bilden, die sogenannte Benzaminsäure muls daher eine anders con- 
stituirte Verbindung sein; setzt man versuchsweise für 1 Aeq. H im Typus Ammoniumoxydhydrat das Radical Benzoyl ein, so findet man in der That, dafs die resultirende Formel 2 Aeq. \# mehr als die der sogenannten Benzaminsäure enthält :

$$
\left.\underset{\mathrm{H}}{\mathrm{NH}_{3}\left(\mathrm{C}_{14} \mathrm{H}_{5} \mathrm{O}_{2}\right)}\right\} \mathrm{O}_{2}=\mathrm{C}_{14} \mathrm{H}_{8} \mathrm{NO}_{4} ; \mathrm{C}_{14} \mathrm{H}_{7} \mathrm{NO}_{4}
$$

Benzaminsăure.

Viel passender würde man defshalb letzlere, um Verwechselungen zu vermeiden, Amidobenzoësäure und die später von mir zu beschreibende Verbindung Biamidobenzoësäure nennen.

Zur Darstellung der Nitrobenzoësäure wandte ich eine von den bisher gebräuchlichen Methoden etwas abweichende an. Die geschmolzene Benzoësäure wurde nämlich in ein Gemisch von zwei Theilen concentrirter Schwefelsüure und einem Theil Salpetersäure (von 1,5 spec. Gew.) in kleinen Portionen eingetragen und dann etwa $\frac{1}{2}$ Stunde gelinde erwärmt; es entsteht dabei keine Binitrobenzoësäure, die überhaupt viel schwieriger zu erhalten ist, als allgemein angegeben wird, sondern W'asser fällt aus dem Säuregemisch nur Nitrobenzoësäure in weilsen Flocken, von deren Reinheit ich mich durch die Analyse des daraus dargestellten Barytsalzes überzeugte.

$0,3285 \mathrm{Grm}$. lufttrockenes Salz verloren bei $100^{\circ} 0,0419$ Grm. Wasser.

$0,3550 \mathrm{Grm}$. bei $100^{\circ}$ getrocknet lieferten $0,1771 \mathrm{Grm}$. schwefelsauren Baryt.

$$
\begin{array}{cccc}
\text { Formel } & & \text { berechnet } & \text { gefunden } \\
\mathrm{C}_{14} \mathrm{H}_{4} \mathrm{BaNO}_{8}+4 \text { aq. } & \text { Wasser } & 13,31 & 12,75 \\
& \text { Baryl } & 32,62 & 32,73
\end{array}
$$

Wird in das Gemisch von Schwefelsäure und Salpetersäure Chlorbenzoyl getropft, so tritt Erwärmung und Entwickelung von Chlor und eines andern, die Augen stark zu 
Thränen reizenden Gases ein; Wasser fällt darauf weifse Flocken, die bei der Analyse sich ebenfalls als reine Nitrobenzoësäure erwiesen.

0,2434 Grm. des gewonnenen Barylsalzes lieferten 0,1205 schwefelsauren Baryt.

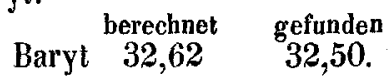

Die Nitrobenzoësäure führte ich auf bekannte Weise durch Kochen mit Schwefelammonium in Amidobenzoësäure über und aus der vom gefällten Schwefel abgegossenen und eingedampften Flüssigkeit schied sich mit concentrirter Salzsäure die salzsaure Amidobenzoësäure in warzenförmig gruppirten Nadeln ab, welche zur Darstellung einiger amidobenzoësaurer Salze diente.

Amidobenzoësaures Natron $\mathrm{C}_{14} \mathrm{H}_{4} \mathrm{Na}\left(\mathrm{NH}_{2}\right)_{4}$ (bei 100 $\mathrm{O}_{4}$ ). - Aus der heifsen wässerigen Lösung des amidobenzoësauren Baryts wurde durch die gerade hinreichende Menge Glaubersalz der Baryt gefällt und das Filtrat bis fast zur Trockne verdunstet. Das Natronsalz ist so leicht in Wasser löslich, dafs es nur schwer gut krystallisirt, welshalb ich die eingedampfte Lösung mit absolutem Alkohol vermischte und den entstandenen weifsen Krystallbrei durch Abpressen zwischen Papier und bei $100^{\circ}$ trocknete; unter dem Mikroscop zeigte er sich aus nadelförmigen Krystallen bestehend.

$0,1291 \mathrm{Grm}$. lieferten 0,0566 schwefelsaures Natron.

$$
\begin{array}{ccc} 
& \text { berechnet } & \text { gefunden } \\
\text { Natron } & 19,50 & 19,13 .
\end{array}
$$

Amidobenzoësaurer Baryt $\mathrm{C}_{14} \mathrm{H}_{4} \mathrm{Ba}\left(\mathrm{NH}_{2}\right) \mathrm{O}_{4}$ (bei $\left.100^{\circ}\right)$. Die in Wasser gelöste salzsaure Amidobenzoësäure wird mit kohlensaurem Baryt bis zum Verschwinden der sauren Reaction digerirt und die heils filtrirte Flüssigkeit im Wasserbade verdunstet; es setzen sich hierauf bei gewisser Concentration grofse, blafsröthlich gefärbte Prismen ab, die durch wiederholtes Umkrystallisiren unter Zusatz von Thierkohle nicht 
vollkommen farblos erhalten werden, aber ein rein weilses Pulver geben.

$0,3955 \mathrm{Grm}$. bei $100^{\circ}$ getrocknet lieferten 0,2234 schwefelsauren Baryt.

0,2709 Grm. bei $100^{\circ}$ getrocknet lieferten beim Verbrennen mit chromsaurem Bleioxyd 0,4084 Kohlensäure und 0,0860 Wasser.

\begin{tabular}{cccc} 
& & berechnet & gefunden \\
$\mathrm{C}_{14}$ & 84 & 41,07 & 41,12 \\
$\mathrm{H}_{6}$ & 6 & 2,93 & 3,51 \\
$\mathrm{Ba}$ & 68,5 & 33,50 & 33,20 \\
$\mathrm{~N}$ & 14 & 6,84 & - \\
$\mathrm{O}_{4}$ & 32 & 15,66 & - \\
\cline { 2 - 4 } & 204,5 & $100,00$. &
\end{tabular}

Amidobenzoësaurer Strontian $\mathrm{C}_{14} \mathrm{H}_{4} \mathrm{Sr}\left(\mathrm{NH}_{2}\right) \mathrm{O}_{4}+2$ aq. Die wässerige Lösung des Barytsalzes wurde mit Schwefelsäure ausgefällt, die vom schwefelsauren Baryt befreile Flüssigkeit mit Strontianhydrat bis zur schwach alkalischen Reaction versetzt und verdunstet. Es schossen schwach röthliche, nach dem Umkrystallisiren aus Alkohol weilse Prismen an, die in Wasser sehr leicht, in Alkohol schwer löslich sind and bei $100^{\circ}$ das Krystallwasser verlieren.

$0,2643 \mathrm{Grm}$. verloren bei $100^{\circ} 0,0232 \mathrm{Grm}$. Wasser und der Rückstand lieferte 0,1223 Grm. schwefelsauren Strontian.

$\begin{array}{lcc} & \text { berechnet } & \text { gefunden } \\ \text { Wasser } & 9,10 & 8,78 \\ \text { Strontian } & \mathbf{2 8 , 8 1} & 28,58 .\end{array}$

Amidobenzoësaurer Kalk $\mathrm{C}_{14} \mathrm{H}_{4} \mathrm{Ca}\left(\mathrm{NH}_{2}\right) \mathrm{O}_{4}$. - Die salzsaure Amidobenzoësäure wurde mit Kalkmilch gekocht und das Filtrat verdunstet. In der concentrirten Flüssigkeit bildeten sich weilse, an der Luft roth werdende, feine Nadeln, die ich nach nochmaligem Umkrystallisiren aus heifsem Weingeist schnell abprelste und bei $100^{\circ}$ trocknete.

0,0863 Grm. lieferten 0,0368 Grm. schwefelsauren Kalk. 


$$
\begin{array}{ccc} 
& \text { berechnet } & \text { gefunden } \\
\text { Kalk } & 17,95 & 17,61 .
\end{array}
$$

Amidobenzoësaure Magnesia $\mathrm{C}_{2} \mathrm{H}_{4} \mathrm{Mg}\left(\mathrm{NH}_{2}\right) \mathrm{O}_{4}+7$ aq. Durch wechselseitige Zersetzung des Barytsalzes und der schwefelsauren Magnesia dargestellt bildet sie grolse, durchsichtige, sechsseitige Säulen mit schiefer Abstumpfungsfläche; sie zersetzt sich rasch an der Luft.

$0,1628 \mathrm{Grm}$. über Schwefelsäure getrocknet verloren bei $100^{\circ} 0,0478 \mathrm{Grm}$. Wasser und der Rückstand lieferte 0,0467 Grm. schwefelsaure Magnesia.

$0,1301 \mathrm{Grm}$. bei $100^{\circ}$ getrocknet lieferten $0,0505 \mathrm{Grm}$. schwefelsaure Magnesia.

\begin{tabular}{lccc} 
& berechnet & \multicolumn{2}{c}{ gefunden } \\
Wasser & 29,86 & 29,36 & - \\
Magnesia & 13,51 & 13,65 & $13,07$.
\end{tabular}

Die Binitrobenzoësäure ist von Cahours durch einstündiges gelindes Erwärmen der Lösung der Benzoësäure in einem Gemisch von Schwefelsäure und Salpetersäure dargestellt; ich erhielt so nur Nitrobenzoësäure oder ein Gemeng von Nitro- und Binitrobenzoësäure und mulste dieses zur Ueberführung in die Binitroverbindung noch sechs Stunden oder länger mit Schwefelsäure und Salpetersäure kochen. Nur zu Anfang der Operation ist die Entwickelung der salpetrigen Säure so heftig, dafs bei nicht gut regulirtem Feuer ein Uebersteigen $z u$ befürchten ist. Später tritt ein Punkt ein, wo keine rothen Dämpfe mehr ausgestofsen werden; lälst man jetzt erkalten, so scheidet sich die Binitrobenzoësäure fast vollständig in glänzenden Krystallen ab; erhilzt man aber länger, so entwickeln sich zum Husten reizende Dämpfe, die Flüssigkeit färbt sich dabei dunkler und auch die beim Erkalten herauskrystallisirende Binitrobenzoësäure ist gefärbt. Die Binitrobenzoësäure ist bei weitem schwerer in kaltem 
und heifsem Wasser löslich, als die Nitrobenzoësäure; von kohlensaurem Natron wird sie mit rother Farbe aufgenommen. Durch Kochen mit Wasser und kohlensaurem Baryt wurde das Barytsalz erhalten, welches sich aus dem heifsen Filtrat beim Erkalten in gelblichen Krystallwarzen absetzte.

$0,1824 \mathrm{Grm}$. bei $100^{\circ}$ getrocknet lieferten $0,0760 \mathrm{Grm}$. schwefelsauren Baryt.

0,3618 Grm. lieferten 0,1497 Grm. schwefelsauren Baryt.

Binitrobenzoësaurer Baryt

$$
\begin{array}{rrr}
\mathrm{C}_{14} \mathrm{H}_{3} \mathrm{Ba}\left(\mathrm{NO}_{4}\right)_{2} \mathrm{O}_{4} \quad \text { Baryt } 27,37 \quad 27,35 \\
& 27,17,
\end{array}
$$

Der Binitrobenzoësäure-Aethyläther entsteht leichl beim Erhitzen der Binitrobenzoësäure mit Weingeist und Schwefelsäure und bildet ölige, beim Erkalten fest werdende Tropfen. In heifsem Weingeist ist er leicht, in kaltem sehr wenig löslich, so dafs die heifs filtrirte weingeistige Lösung zu einem aus langen, seideglänzenden Nadeln bestehenden Magma erstarrt. - Ich versuchte, durch Digestion dieses Aethers mit Schwefelammonium das Biamidobenzamid darzustellen; es schied sich auch Schwefel in reichlicher Menge ab und beim Verdunsten des Filtrats blieb eine krystallinische Masse, die aber zur Analyse und Prüfung der Eigenschaften nicht rein genug zu bekommen war.

Leicht liefs sich dagegen der Aether in das Binitrobenzamid durch mehrtägige Digestion mit weingeistigem Ammoniak verwandeln; in diesem löste er sich mit blutrother Farbe und nach der Concentration setzten sich schwach gelblich gefärbte fettglänzende Säulen und Blättchen des Amids ab. - In kaltem Wasser ist das Binitrobenzamid wenig, in heilsem etwas mehr löslich, die Lösung schmeckt bitter und reagirt neutral. Es schmilzt bei $183^{\circ}$ und zersetzt sich in höherer Temperatur, ohne zu sublimiren. Die ammoniakalische Lösung giebt mit salpetersaurem Silberoxyd keinen Niederschlag. 
Zur Bestimmung des Stickstoffs, welcher nicht in Form von Untersalpetersäure in dem Binitrobenzamid enthalten ist, wurde eine gewogene Menge mit Kalilauge in einem Retörtchen erhitzt und das entweichende Ammoniak in titrirter Schwefelsäure aufgefangen. Die Verbrennung wurde mit chromsaurem Bleioxyd ausgeführt, lieferte aber etwas zu viel Kohlenstoff, weil vielleicht Untersalpetersäure in den Kaliapparat gelangte.

$0,0918 \mathrm{Grm}$. lieferten mit Kalilauge gekocht $\mathbf{0 , 0 0 6 3 3 6}$ Grm. Stickstoff; 0,1737 Grm. lieferten 0,2604 Grm. Kohlensäure und 0,0448 Grm. Wasser.

$$
\text { Die Formel } \mathrm{N}\left\{\begin{array}{l}
\mathrm{C}_{14}^{\mathrm{C}_{3}} \mathrm{H}_{\mathbf{H}}^{\mathrm{H}}\left(\mathrm{NO}_{4}\right)_{2} \mathrm{O}_{2} \\
\underset{H}{H}
\end{array}\right. \text { verlangt : }
$$

\begin{tabular}{|c|c|c|c|}
\hline$C_{14}$ & 84 & $\begin{array}{c}\text { berechnet } \\
39,81\end{array}$ & $\begin{array}{c}\text { gefunden } \\
40,87\end{array}$ \\
\hline $\mathrm{H}_{5}$ & 5 & 2,37 & 2,88 \\
\hline $2 \mathrm{NO}_{4}$ & 92 & 43,60 & - \\
\hline $\mathrm{N}$ & 14 & 6,63 & 6,90 \\
\hline $\mathrm{O}_{2}$ & 16 & 7,59 & - \\
\hline
\end{tabular}

Um die Binitrobenzoësäure in Biamidobenzoësäure überzuführen, leitete ich durch die heilse ammoniakalische Lösung anhaltend Schwefelwasserstoff, wobei dieselben Erscheinungen eintraten, welche man bei Darstellung der übrigen Amidverbindungen nach dieser Methode beobachtet. Die vom ausgeschiedenen Schwefel filtrirte Lösung wurde im Wasserbade eingedampft, mit Salzsäure übersättigt und noch heifs filtrirt; aus dem Filtrat setzten sich nach einiger Zeit grüne Krystalle von salzsaurer Biamidobenzoësäure ab.

Die durch Digestion der schwefelsauren Verbindung in wässeriger Lösung mit kohlensaurem Baryt und Verdunsten des farblosen Filtrats zuerst im Wasserbade, später über Schwefelsäure in Form grünlich gefärbter kleiner spiefsiger 
Krystalle erhaltene Biamidobenzoësäure ist in Wasser leicht und auch in Alkohol und Aether löslich, ohne Geschmack und Reaclion auf Pflanzenfarben, schmilzt und schwärzt sich bei etwa $195^{\circ}$ und läfst sich nicht sublimiren.

Bemerkenswerth ist die Eigenschaft der Biamidobenzoësäure, mit Basen keine, wohl aber mit Säuren gut krystallisirende Verbindungen einzugehen, wefshalb ihr der Name einer Säure mit Unrecht zukömmt. Es zeigt sich hier ein ähnliches Verhalten, wie es schon im ausgezeichneten Grade beim Anilin beobachtet wurde, dafs nämlich die Substitutionsproducte in der Regel den Hauptcharacter der Verbindung, von welcher sie sich ableiten, beibehalten, der eintretende Stoff aber doch seinen chemischen Character im Substitutionsproduct nicht vollständig verläugnet und demselben in dem Mafse mehr aufprägt, als die Zahl der durch ihn vertretenen Wasserstoffäquivalente zunimmt. Die Benzoësäure ist als starke Säure bekannt; mit Eintritt eines Aequivalents des basische Eigenschaften bedingenden Amids zeigen sich auch schon basische Eigenschaften, denn wir sehen die Amidobenzoësäure nicht nur Verbindungen mit Basen, sondern auch mit Säuren eingehen; mit Eintritt eines zweiten Aequivalents Amid gehen die Eigenschaften einer Säure vollständig verloren, da die Biamidobenzoësäure nur noch mil Säuren Salze bildet.

Salzsaure Biamidobenzoësäzure $\mathrm{C}_{14} \mathrm{H}_{4}\left(\mathrm{AH}_{2}\right)_{2} \mathrm{O}_{4}, 2 \mathrm{HGl}$. Die noch unreine Verbindung, wie man sie bei Bereitung der Biamidobenzoësäure bekomml, läfst sich schwierig durch Umkrystallisiren reinigen; leichter gelangt man zum Ziele, wenn man die Lösung in wenig Wasser mit concentrirter Salzsäure vermischt; es scheiden sich dadurch entweder sogleieh weilse Nadeln $a b$, oder es entsteht erst ein flockiger Niederschlag, der sich aber bald in dieselben Nadeln umsetzt. Sollte das Salz noch nicht vollkommen rein sein, so wiederholt 
man noch einmal dieselbe Operation. Die salzsaure Biamidobenzoësäure ist sehr leicht in Wasser und auch in Weingeist und Aether löslich; die wässerige Lösung zersetzt sich beim Stehen an der Luft unter Abscheidung schwarzer Flocken. Die trockene Verbindung schmilzt beim Erhitzen, zersetzt sich dann und liefert ein aus Salmiak bestehendes Sublimat. Zur Analyse wurde das zwischen Papier abgeprefste Salz erst über Schwefelsäure, dann bei $60^{\circ}$ getrocknet.

$0,2469 \mathrm{Grm}$. lieferten 0,3095 Grm. Chlorsilber.

$0,2267 \mathrm{Grm}$. lieferten $0,2849 \mathrm{Grm}$. Chlorsilber.

berechnet gefunden

Chlor $\quad 31,55 \quad 31,02 \quad 31,10$.

Platinchlorid bringt in der wässerigen Lösung keinen Niederschlag hervor, der sich auch nicht beim Vermischen mil Weingeist und Aether bildet; läfst man die Mischung jedoch über Schwefelsäure verdunsten, so setzen sich braun gefärbte Krusten ab, die nach dem Trocknen ein grauweifses Pulver geben. Die Zusammenselzung scheint $\mathrm{C}_{14} \mathrm{H}_{4}\left(\mathrm{NH}_{2}\right)_{2} \mathrm{O}_{4}$, $2 \mathrm{HEl}, \mathrm{PtCl}_{2}$ zu sein, denn $0,0443 \mathrm{Grm}$. hinterliefsen nach dem Glühen $0,0110 \mathrm{Grm} .=24,83 \mathrm{pC}$. Platin und obige Formel verlangt 25,01 pC. Platin. In diesem Doppelsalze mülsten 2 Aeq. Platinchlorid vorkommen, wenn es mit den entsprechenden Salzen der übrigen Basen, welche 2 Aeq. Säure sältigen (Chinin, Cinchonin), im Einklang stände.

Schwefelsaure Biamidobenzoësäure $\mathrm{C}_{14} \mathrm{H}_{4}\left(\mathrm{NH}_{1}\right]_{2} \mathrm{O}_{4}, \mathrm{~S}_{2} \mathrm{H}_{2} \mathrm{O}_{8}$. - Die salzsaure Verbindung wurde in verdünnter Schwefelsäure gelöst und die Lösung concentrirt; die angeschossenen, noch bräunlich gefärbten Tafeln und Blätter wurden durch Umkrystallisiren aus Weingeist fast farblos erhalten. Sie sind leicht in Wasser, etwas schwerer in Weingeist löslöch; die Lösungen sind sehr leicht zersetzbar und erscheinen im durchfallenden Lichte bräunlichgelb, im reflectirten 
grasgrün. Zur Analyse wurden die Krystalle über Schwefelsäure getrocknet.

0,3560 Grm. lieferten 0,3346 Grm. schwefelsauren Baryt.

0,4777 Grm. lieferten 0,4543 Grm. schwefelsauren Baryt.

Beim Erhitzen mit Natronkalk und Auffangen des Ammoniaks in titrirter Schwefelsäure lieferten

0,1709 Grm. Substanz 0,019296 Grm. Stickstoff.

0,1079 Grm. Substanz 0,011808 Grm. Stickstoff.

Die mit chromsaurem Bleioxyd ausgeführte Verbrennung gab von $0,1634 \mathrm{Grm}$. Substanz 0,2056 Grm. Kohlensáure und $0,0687 \mathrm{Grm}$. Wasser; von 0,1216 Grm. Substanz 0,1498 Grm. Kohlensäure und 0,0541 Grm. Wasser.

\begin{tabular}{lrrrr} 
& & berechnet & \multicolumn{2}{c}{ gefunden } \\
$\mathrm{C}_{14}$ & 84 & 33,60 & 34,33 & 33,55 \\
$\mathrm{H}_{10}$ & 10 & 4,00 & 4,65 & 4,93 \\
$\mathrm{~N}_{2}$ & 28 & 11,20 & 11,29 & 10,94 \\
$\mathrm{O}_{6}$ & 48 & 19,20 & - & - \\
$\mathrm{S}_{2} \mathrm{O}_{6}$ & 80 & 32,00 & 32,25 & 32,68 \\
\hline & 250 & $100,00$. & &
\end{tabular}

Salpetersaure Biamidobenzoësäure, durch wechselseilige Zersetzung aus der schwefelsauren Verbindung und salpetersaurem Baryt erhalten, schiefst in dunkel gefärbten Krystallen an.

Essigsaure Biamidobenzoësäure stellte ich durch Ausfällung der schwefelsauren Verbindung mit essigsaurem Baryt dar. Die Lösung zersetzle sich beim Abdampfen unter Abscheidung eines braunen Pulvers, bei weiterem Verdunsten der filtrirten Fliissigkeit krystallisirten jedoch bräunlich gefärbte Prismen heraus.

Oxalsaure Biamidobenzoësäure bildet braune Nadeln, die beim Verdunsten der salzsauren Verbindung mit Oxalsäure entstehen. 
Ein Versuch, die Aethyl-Biamidobenzoësäure durch Erhitzen der Biamidobenzoësäure mit Jodäthyl im zugeschmolzenen Rohre auf $100^{\circ}$ darzustellen, gab ein negatives Resultat; die Masse wurde fest und lieferte auf Zusatz von Salzsäure zur wässerigen Lösung weifse Nadeln, welche bei der Analyse sich als salzsaure Biamidobenzoësäure erwiesen.

Auch durch Zersetzung der Biamidobenzoësäure in heifser wässeriger Lösung mit salpetriger Säure, welche nach Gerland's Versuchen die Amidobenzoësäure in Oxybenzoësäure üherführt, wurde nur eine rothe harzige, nicht krystallisirbare Masse erhalten, obgleich ich das Einleiten des Gases mehrere Tage lang fortsetzte.

Analysen vom Laumontit aus dem Sarnthal bei Botzen in Tyrol;

\section{von $H$. Gericke.}

Auf einer Reise in Tyrol fand ich im Sarnthale bei Botzen, welches sich besonders durch seine schönen Porphyre auszeichnet, ein Mineral, welches, wie aus folgenden Untersuchungen hervorgeht, Laumontit war. Bis jetzt ist, soviel ich weifs, dieser Fundort noch nicht bekannt gewesen.

Der Laumontit kommt daselbst sowohl blätterig als erdig vor. Die Krystalle sind höchstens 2 bis 3 Linien Iang und 1 bis $1 \frac{1}{2}$ Linien dick, und aus deren Spaltungsflächen, welche man ganz deutlich erkennen kann, ergab sich, dafs dieses Mineral auch dem monoklinoëdrischen Krystallsystem (Breithaupt) angehört. Es kommt in vierseitigen Prismen von röthlicher Farbe vor und ist sehr leicht zerbröcklich, in Folge 